\title{
Caracterización sociodemográfica, clínica y terapéutica de pacientes con falla cardiaca con fracción de eyección intermedia: cohorte MED-ICAi
}

\author{
Sociodemographic, clinical and therapeutic characteristics of patients with heart failure \\ with mid-range ejection fraction: MED-ICAi cohort
}

\author{
Kevin Y. Ruales-Mora ${ }^{1,2 *}$, Tatiana Rojas-López ${ }^{1}$, Julio C. Valencia-Maturana ${ }^{1}$, Irene Salas-Giraldo', \\ Luis M. Ruiz-Peña ${ }^{1}$, Marly García-Sánchez ${ }^{3}$ y Andrés Zapata-Cárdenas ${ }^{1,4}$ \\ ${ }^{1}$ Departamento de Medicina Interna, Universidad de Antioquia; ${ }^{2}$ Departamento de Medicina Interna, IPS Universitaria Clínica León XIII; ${ }^{3}$ Facultad \\ de Salud, Universidad Pontificia Bolivariana; ${ }^{4}$ Departamento de Medicina Interna, Hospital Universitario San Vicente Fundación. Medellín, Colombia
}

\section{Resumen}

\begin{abstract}
Antecedentes: Clásicamente, la falla cardiaca se ha clasificado en dos grupos, según tengan fracción de eyección preservada o reducida; no obstante, en fecha reciente se ha añadido el grupo de fracción intermedia, y aún existe gran desconocimiento sobre sus características fisiopatológicas y clínicas. Objetivo: Caracterizar el grupo de pacientes con fracción intermedia en cuanto a sus variables sociodemográficas, clínicas y de tratamiento. Método: Se realizó un estudio descriptivo, retrospectivo, en el que se analizaron historias clínicas de pacientes con falla cardiaca hospitalizados por agudización en el periodo comprendido entre enero de 2015 y diciembre de 2017. Resultados: Se revisaron 1536 historias clínicas, de las cuales 864 cumplían los criterios de inclusión. El grupo con fracción intermedia correspondió a 83 (9.6\%) pacientes, en quienes se encontró predominio del sexo femenino (53\%) y una edad mediana de 77 años. La coronariopatía fue la etiología más frecuente (26.5\%), mientras que la falta de adherencia a los medicamentos fue la causa principal de descompensación (14.5\%). Los medicamentos más usados fueron los betabloqueadores y la furosemida, tanto al ingreso como al egreso. El grupo con mayor mortalidad fue el de fracción de eyección reducida (4.1\%). La estancia hospitalaria, el ingreso y la estancia en la unidad de cuidados intensivos fueron similares en todos los grupos, independientemente de la fracción de eyección. Conclusiones: Los hallazgos son similares a los descritos en otras poblaciones internacionales y en algunas nacionales, y avalan la hipótesis de un fenotipo intermedio con un comportamiento etiológico semejante al de la fracción de eyección reducida.
\end{abstract}

Palabras clave: Insuficiencia cardiaca. Volumen sistólico. Mortalidad. Factores de riesgo.

\section{Abstract}

Background: Classically, heart failure has been classified in two groups, depending on a preserved or reduced ejection fraction, but a mid-range ejection fraction group has been introduced recently, and there is still great ignorance about its physiopathological and clinical characteristics. Objective: To characterize this group of patients as for their sociodemographic, clinical and treatment variables. Method: We carried out a descriptive, retrospective study, by analyzing medical records from

\section{Correspondencia:}

*Kevin Y. Ruales-Mora E-mail: valyam1989@gmail.com
Disponible en internet: 29-07-2021 Rev Colomb Cardiol. 2021;28(3):254-262 www.rccardiologia.com 0120-5633 / @ 2020 Sociedad Colombiana de Cardiología y Cirugía Cardiovascular. Publicado por Permanyer. Este es un artículo open access bajo la licencia CC BY-NC-ND (http://creativecommons.org/licenses/by-nc-nd/4.0/). 
patients hospitalized with acute heart failure between January 2015 and December 2017. Results: We reviewed 1536 medical records of which 864 met the inclusion criteria. The mid-range ejection fraction group corresponded to $83(9.6 \%)$ of patients, of which the majority were women (53\%), with a median age of 77 years, coronary heart disease as the most frequent etiology (26.5\%) and lack of adherence to medications as the main cause of decompensation (14.5\%). The most frequently used drugs were betablockers and furosemide, both upon admission and discharge. Mortality was higher between patients with reduced ejection fraction (4.1\%). Hospital stay, admission to and length of stay in an ICU, were similar between all groups regardless of ejection fraction. Conclusions: Our findings are similar to those described in previous international and national cohorts, and support the hypothesis of an intermediate phenotype with an etiology similar to that seen with a reduced ejection fraction.

Key words: Heart failure. Stroke volume. Mortality. Risk factors.

\section{Introducción}

La falla cardiaca es altamente prevalente, constituye un problema de salud pública y es una de las principales causas de hospitalización en mayores de 65 años ${ }^{1,2}$. Clásicamente se ha dividido en dos grupos en función de la fracción de eyección del ventrículo izquierdo (FEVI): falla cardiaca con FEVI preservada y falla cardiaca con FEVI reducida ${ }^{3}$.

En el año 2014, las guías internacionales del American College of Cardiology y la American Heart Association identificaron un nuevo grupo intermedio, con FEVI entre el $40 \%$ y el $50 \%^{4}$, debido a que en los estudios de FEVI reducida se tomó como punto de corte $<35-40 \%$ y en los de FEVI preservada se tomó $>45 \%{ }^{1}$. Esta nueva categoría no fue creada con bases fisiopatológicas ni datos sobre desenlaces específicos, sino por la necesidad de dar nombre al grupo conocido como "área gris» 5 .

Esta zona, creada por la arbitrariedad en los puntos de corte, representa aproximadamente el $10-20 \%$ de todos los pacientes con falla cardiaca ${ }^{6}$. Aún se ignora si tienen características propias o si pueden pasar a ser parte de uno de los otros dos grupos. Hasta ahora, existen artículos que sugieren que sus características clínicas, ecocardiográficas, hemodinámicas y bioquímicas se encuentran en un punto medio entre la FEVI preservada y la FEVI reducida ${ }^{5}$.

Un estudio colombiano, en el subgrupo de FEVI intermedia, describe una edad mayor, una estancia hospitalaria más prolongada y un ingreso considerable a la unidad de cuidados intensivos (UCl); ; sin embargo, la cantidad escasa de pacientes limita el análisis.

En Medellín se realizó un estudio descriptivo, pionero en la caracterización de los pacientes con falla cardiaca y FEVI intermedia. Se trazaron como objetivos principales describir las características epidemiológicas, clínicas y de tratamiento de estos pacientes, y hacer una comparación con los grupos con FEVI preservada y reducida.

\section{Método}

\section{Diseño del estudio}

Se realizó un estudio retrospectivo con una base de datos generada a partir de registros clínicos de la IPS Universitaria Clínica León XIII de Medellín, Colombia. Se revisaron las historias clínicas del periodo comprendido entre enero de 2015 y diciembre de 2017 con el código diagnóstico CIE 10 de insuficiencia cardiaca (I50X).

\section{Participantes}

Se incluyeron pacientes mayores de 18 años que ingresaron a la institución por falla cardiaca agudamente descompensada, entendida como el comienzo o el cambio rápido en los signos y síntomas de la enfermedad, que requerían un tratamiento urgente. Para los pacientes que ingresaron en más de una ocasión a la institución durante el periodo de estudio solo se tuvo en cuenta el primer ingreso. Se excluyeron las mujeres gestantes y los pacientes que no requirieron hospitalización o que no tuvieron ecocardiograma intrahospitalario ni extrahospitalario para la clasificación del estadio según la FEVI.

\section{Tamaño del estudio}

Dado que se trató de un estudio descriptivo, para lograr una adecuada caracterización de la población se intentó recolectar la mayor muestra de pacientes durante el tiempo asignado. No se realizó muestreo probabilístico, sino que se tomó una muestra a conveniencia. 


\section{Variables}

Se revisaron en el Sistema de Gestión Hospitalaria IPS - GHIPS todas las historias clínicas filtradas con el código diagnóstico CIE-10 de falla cardiaca (I50X) en el periodo de estudio. Como variables sociodemográficas y de historia personal se tomaron la edad en años, el sexo, la clasificación de la New York Heart Association (NYHA) de base y al ingreso (clases I a IV), los medicamentos utilizados al ingreso y al alta, y la presencia o no de comorbilidad.

Del episodio estudiado se describieron como variables clínicas el síntoma principal de consulta, el perfil hemodinámico según la clasificación de Stevenson, los signos vitales al ingreso, el tiempo de evolución del cuadro clínico, la etiología de la falla cardiaca, el requerimiento de $\mathrm{UCI}$, la duración de estancia hospitalaria y en la $\mathrm{UCl}$, y la mortalidad intrahospitalaria. Se precisó la etiología de la descompensación cuando fue documentada.

Para la clasificación del estadio según la FEVI, se registró la fracción de eyección ambulatoria y se tomaron los datos del ecocardiograma intrahospitalario. En caso de no tener ecocardiograma intrahospitalario se utilizó la FEVI documentada previamente. Se revisaron las características ecocardiográficas, como la presencia de disfunción diastólica, hipertensión pulmonar y valvulopatía. Se hizo el diagnóstico de falla cardiaca con FEVI preservada según la clínica compatible y las alteraciones estructurales 0 funcionales en el ecocardiograma.

La recolección de la información estuvo a cargo de los investigadores por medio de un formato de captura de datos diseñado en Microsoft Office Excel囚. Los datos se procesaron con el software SPSS versión 22.0.

\section{Análisis estadístico}

Se calcularon las frecuencias absoluta y relativa de las variables cualitativas. Se utilizaron medidas de tendencia central y medidas de variabilidad (promedio, mediana y rango intercuartílico [RIC]) para las variables cuantitativas. Se describió la mortalidad intrahospitalaria para toda la muestra.

\section{Consideraciones éticas}

No se realizó ninguna intervención ni modificación intencionada de aspectos fisiológicos, psicológicos o sociales de la población estudiada, por lo que no hubo lugar a daño o perjuicio de la integridad de los participantes, lo que justifica la no obtención del consentimiento informado. El proyecto de investigación fue aprobado, previamente a su ejecución, por el comité de ética médica de la institución, y fue financiado en su totalidad por los autores.

\section{Resultados}

\section{Pacientes}

La figura 1 ilustra la distribución de la muestra obtenida. La búsqueda inicial arrojó 1536 historias clínicas de pacientes, de las cuales 297 correspondían a duplicados por reingresos (1239 historias elegibles) y 381 no cumplían los criterios de inclusión por no tener ecocardiograma o no cursar con agudización de la falla cardiaca. Finalmente, 864 adultos fueron incluidos en el estudio y analizados.

Los pacientes se clasificaron según su FEVI y se obtuvieron cuatro grupos: FEVI $40-49 \%$ (intermedia) con $83(9.6 \%)$ pacientes, $\mathrm{FEVI}<40 \%$ (reducida) con $338(39.1 \%)$ pacientes, FEVI > 50\% (preservada) con 289 (33.4\%) pacientes, y un grupo de 154 (17.8\%) pacientes sin ecocardiograma intrahospitalario que se subdividieron según la FEVI extrahospitalaria.

\section{Características sociodemográficas y de historia personal}

Del total de los pacientes evaluados, 470 (54.4\%) eran mujeres y 394 (45.6\%) eran hombres. En el grupo con FEVI intermedia predominó el sexo femenino (53\%), proporción que se mantuvo en quienes tenían FEVI preservada y se invirtió en los de FEVI reducida. La mediana de edad fue de 75 años (rango: 18-105 años), con una distribución semejante en los tres grupos (Tabla 1).

La comorbilidad más frecuente incluyó hipertensión arterial (32.1\%), enfermedad pulmonar obstructiva crónica $(14.8 \%)$, diabetes mellitus tipo $2(14 \%)$, infarto agudo de miocardio (10.6\%) y fibrilación auricular (9.8\%). En los pacientes con FEVI intermedia, las enfermedades más prevalentes fueron hipertensión arterial, fibrilación auricular, diabetes mellitus y síndrome coronario, con una distribución semejante a la de los otros dos grupos; sin embargo, fue mayor la coexistencia de fibrilación auricular (Tabla 1).

En los pacientes con FEVI intermedia, la clasificación NYHA basal fue clase II en el $50.6 \%$, y al ingreso la más frecuente fue la clase III (48.2\%), seguida de la IV 


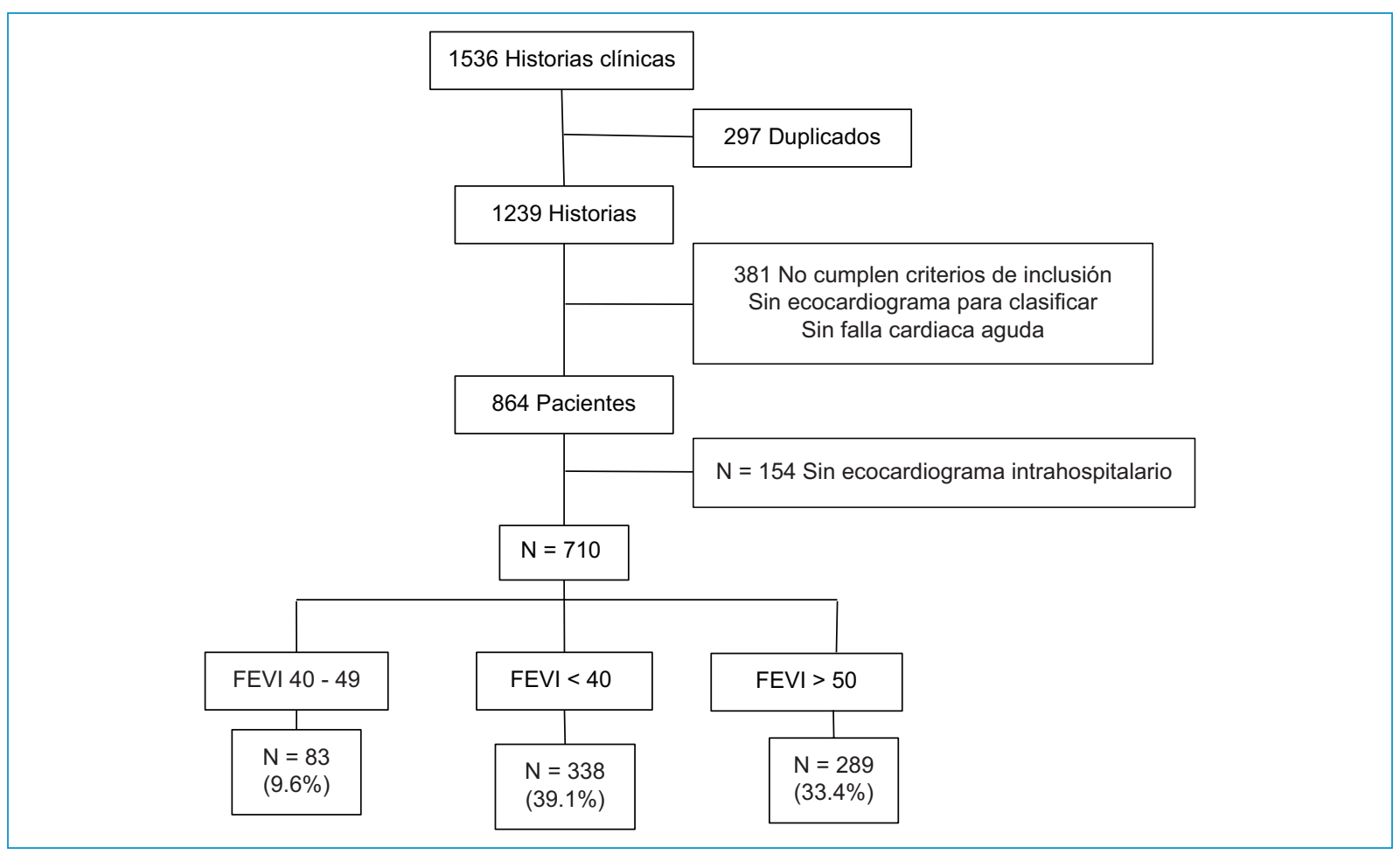

Figura 1. Distribución de la muestra. FEVl: fracción de eyección del ventrículo izquierdo.

(39.8\%). En este parámetro no hay diferencias respecto a la cohorte total ni a los otros grupos (Tabla 1).

\section{Variables clínicas}

El perfil hemodinámico predominante fue Stevenson B (91.6\% para el grupo de FEVI intermedia), con una pequeña proporción de Stevenson A y C $(4.8 \%$ y $3.6 \%$, respectivamente) y ningún paciente con Stevenson L; presentación muy similar a lo encontrado para los pacientes con FEVI preservada y reducida (Tabla 2).

En los pacientes con FEVI intermedia, la etiología más común fue la coronariopatía (26.5\%), seguida de causa no determinada $(24.1 \%)$ y, en tercer lugar, de hipertensión arterial (12\%), con un comportamiento similar al de los pacientes con FEVI reducida (coronariopatía en el $29.3 \%$ ), mientras que en aquellos con FEVI preservada prevaleció la hipertensión arterial (29.1\%). En el total de pacientes, la causa idiopática fue del $7.2 \%$ y hasta un $23.5 \%$ de los pacientes egresaron con una etiología indeterminada (Tabla 3).

Las principales causas registradas de descompensación en los pacientes con FEVI intermedia fueron la no adherencia al tratamiento (14.5\%), las arritmias (13.3\%) y las infecciones (9.6\%). No se registró la etiología en el $30.1 \%$. En los tres grupos, la no adherencia fue una de las principales causas. La enfermedad coronaria contribuyó a la agudización en los pacientes con FEVI intermedia y reducida, en un $8.4 \%$ y un $7.1 \%$, respectivamente, mientras que en aquellos con FEVI preservada solo se observó en un $2.1 \%$ (Tabla 3).

\section{Tiempo de evolución, estancia}

hospitalaria, ingreso a la UCl y mortalidad general

El tiempo de evolución del cuadro clínico tuvo una mediana de 7 días (RIC: 3-15), igual para los tres grupos. La mediana de estancia hospitalaria para el total de los pacientes fue de 6 días (RIC: 4-9); el grupo con FEVI intermedia tuvo una mediana de 7 días (RIC: 5-10) (Tabla 3).

Del total de los pacientes, 23 (3.2\%) requirieron ingreso a la UCI. En el grupo con FEVI intermedia, 2 (2.4\%) pacientes requirieron ingreso a la $\mathrm{UCl}$ y la mediana de estancia fue de 5 días (RIC: 1-9) (Tabla 3).

Fallecieron 23 pacientes (3.2\%), de los cuales solo $1(1.2 \%)$ pertenecía al grupo de FEVI intermedia; de este se sabe que la etiología de la falla fue una coronariopatía, ingresó con un perfil hemodinámico Stevenson By no se documentóla causa de la descompensación. La mortalidad fue más alta en los pacientes con FEVI 
Rev Colomb Cardiol. 2021;28(3)

Tabla 1. Variables sociodemográficas y de historia personal

\begin{tabular}{|c|c|c|c|c|}
\hline \multirow[t]{2}{*}{ Variable } & FEVI $<40 \%$ & FEVI $40-49 \%$ & FEVI $>50 \%$ & Total \\
\hline & 338 (39.1\%) & $83(9.6 \%)$ & $289(33.4 \%)$ & $710(100 \%)$ \\
\hline Sexo femenino & $141(41.7 \%)$ & $44(53 \%)$ & $200(69.2 \%)$ & $385(54.2 \%)$ \\
\hline Edad (años) & $73(20-96)$ & 77 (23-105) & 76 (18-98) & 75 (18-105) \\
\hline $\begin{array}{l}\text { Clase NYHA base } \\
\text { I } \\
\text { II } \\
\text { III } \\
\text { IV } \\
\text { No reportado }\end{array}$ & $\begin{array}{c}69(20.4 \%) \\
161(47.6 \%) \\
78(23.1 \%) \\
19(5.6 \%) \\
11(3.3 \%)\end{array}$ & $\begin{array}{c}18(21.7 \%) \\
42(50.6 \%) \\
16(19.3 \%) \\
6(7.2 \%) \\
1(1.2 \%)\end{array}$ & $\begin{array}{c}51(17.6 \%) \\
141(48.8 \%) \\
63(21.8 \%) \\
22(7.6 \%) \\
12(4.2 \%)\end{array}$ & $\begin{array}{c}138(19.4 \%) \\
344(48.5 \%) \\
157(22.1 \%) \\
47(6.6 \%) \\
24(3.4 \%)\end{array}$ \\
\hline $\begin{array}{l}\text { Clase NYHA ingreso } \\
\text { I } \\
\text { II } \\
\text { III } \\
\text { IV } \\
\text { No reportado }\end{array}$ & $\begin{array}{c}5(1.5 \%) \\
26(7.7 \%) \\
145(42.9 \%) \\
156(46.2 \%) \\
6(1.8 \%)\end{array}$ & $\begin{array}{c}1(1.2 \%) \\
7(8.4 \%) \\
40(48.2 \%) \\
33(39.8) \\
2(2.4 \%)\end{array}$ & $\begin{array}{c}6(2.1 \%) \\
31(10.7 \%) \\
120(41.5 \%) \\
122(42.2 \%) \\
10(3.5 \%)\end{array}$ & $\begin{array}{c}12(1.7 \%) \\
64(9.0 \%) \\
305(43.0 \%) \\
311(43.8 \%) \\
18(2.5 \%)\end{array}$ \\
\hline $\begin{array}{l}\text { Principal comorbilidad } \\
\text { Diabetes mellitus } \\
\text { Enfermedad pulmonar obstructiva crónica } \\
\text { Enfermedad renal crónica } \\
\text { Fibrilación auricular } \\
\text { Hipertensión arterial } \\
\text { Enfermedad coronaria }\end{array}$ & $\begin{array}{c}41(12.1 \%) \\
44(13 \%) \\
7(2.1 \%) \\
30(8.9 \%) \\
118(34.9 \%) \\
38(11.2 \%)\end{array}$ & $\begin{array}{c}12(14.5 \%) \\
6(7.2 \%) \\
2(2.4 \%) \\
13(15.7 \%) \\
27(32.5 \%) \\
10(12 \%)\end{array}$ & $\begin{array}{c}50(17.3 \%) \\
60(20.8 \%) \\
4(1.4 \%) \\
21(7.3 \%) \\
98(33.9 \%) \\
15(5.2 \%)\end{array}$ & $\begin{array}{c}103(14.5 \%) \\
110(15.5 \%) \\
13(1.8 \%) \\
64(9.0 \%) \\
243(34.2 \%) \\
63(8.9 \%)\end{array}$ \\
\hline
\end{tabular}

FEVI: fracción de eyección del ventrículo izquierdo; NYHA: New York Heart Association.

Tabla 2. Variables clínicas: principales síntomas de consulta y perfil hemodinámico

\begin{tabular}{|c|c|c|c|c|}
\hline Variable & $\begin{array}{l}\text { FEVI < 40\% } \\
338(39.1 \%)\end{array}$ & $\begin{array}{l}\text { FEVI } 40-49 \% \\
83(9.6 \%)\end{array}$ & $\begin{array}{l}\text { FEVI > 50\% } \\
289(33.4 \%)\end{array}$ & $\begin{array}{c}\text { Total } \\
710(100 \%)\end{array}$ \\
\hline $\begin{array}{l}\text { Síntoma principal de consulta } \\
\text { Disnea } \\
\text { Dolor de tórax } \\
\text { Edema } \\
\text { Fatiga } \\
\text { Otros } \\
\text { Palpitaciones } \\
\text { Tos }\end{array}$ & $\begin{array}{c}203(60.1 \%) \\
37(10.9 \%) \\
71(21 \%) \\
10(3 \%) \\
12(3.6 \%) \\
3(0.9 \%) \\
2(0.6 \%)\end{array}$ & $\begin{array}{c}57(68.7 \%) \\
8(9.6 \%) \\
12(14.5 \%) \\
0(0 \%) \\
5(6 \%) \\
1(1.2 \%) \\
0(0 \%)\end{array}$ & $\begin{array}{c}160(55.4 \%) \\
29(10 \%) \\
82(28.4 \%) \\
6(2.1 \%) \\
9(3.1 \%) \\
3(1 \%) \\
0(0 \%)\end{array}$ & $\begin{array}{c}420(59.2 \%) \\
74(10.4 \%) \\
165(23.2 \%) \\
16(2.3 \%) \\
26(3.7 \%) \\
7(1.0 \%) \\
2(0.3 \%)\end{array}$ \\
\hline $\begin{array}{l}\text { Perfil hemodinámico (Stevenson) } \\
\text { A } \\
\text { B } \\
\text { C } \\
\text { L }\end{array}$ & $\begin{array}{c}13(3.8 \%) \\
312(92.3 \%) \\
12(3.6 \%) \\
1(0.3 \%)\end{array}$ & $\begin{array}{c}4(4.8 \%) \\
76(91.6 \%) \\
3(3.6 \%) \\
0(0 \%)\end{array}$ & $\begin{array}{c}13(4.5 \%) \\
273(94.5 \%) \\
3(1 \%) \\
0(0 \%)\end{array}$ & $\begin{array}{c}30(4.2 \%) \\
661(93.1 \%) \\
18(2.5 \%) \\
1(0.1 \%)\end{array}$ \\
\hline
\end{tabular}

FEVI: fracción de eyección del ventrículo izquierdo.

reducida y preservada $(4.1 \%$ y $2.8 \%$, respectivamente) (Tabla 3).

En cuanto a la estancia hospitalaria y la mortalidad de acuerdo con el perfil hemodinámico presentado, los pacientes con Stevenson $C$ tuvieron una mediana de hospitalización de 10 días (RIC: 7.25-16.25), los del grupo de Stevenson A de 8 días (RIC: 4.25-9.5) y los de Stevenson B de 7 días (RIC: 5-10). En cuanto a la mortalidad, la más alta se presentó en el grupo de Stevenson B (82.9\%), con un $45 \%$ que correspondían al grupo de FEVI reducida y un $7 \%$ al grupo de FEVI intermedia. 
Tabla 3. Variables clínicas: etiología de la falla cardiaca y de la descompensación, estancia hospitalaria y en la unidad de cuidados intensivos, y mortalidad intrahospitalaria

\begin{tabular}{|c|c|c|c|c|}
\hline Variable & $\begin{array}{l}\text { FEVI < 40\% } \\
338(39.1 \%)\end{array}$ & $\begin{array}{l}\text { FEVI } 40-49 \% \\
83(9.6 \%)\end{array}$ & $\begin{array}{l}\text { FEVI > 50\% } \\
289(33.4 \%)\end{array}$ & $\begin{array}{c}\text { Total } \\
710(100 \%)\end{array}$ \\
\hline Tiempo de evolución del cuadro clínico (RIC) & $7(4-15)$ & $7(3-15)$ & $7(4-15)$ & $7(3-15)$ \\
\hline $\begin{array}{l}\text { Etiología de la falla cardiaca } \\
\text { Arritmia } \\
\text { Miocardiopatía } \\
\text { Coronariopatía } \\
\text { Hipertensión arterial } \\
\text { Idiopática } \\
\text { Otros } \\
\text { Etiología no determinada } \\
\text { Tóxico } \\
\text { Valvulopatía }\end{array}$ & $\begin{array}{c}17(5 \%) \\
17(5 \%) \\
99(29.3 \%) \\
57(16.9 \%) \\
28(8.3 \%) \\
22(6.5 \%) \\
68(20.1 \%) \\
11(3.3 \%) \\
19(5.6 \%)\end{array}$ & $\begin{array}{c}8(9.6 \%) \\
3(3.6 \%) \\
22(26.5 \%) \\
10(12 \%) \\
6(7.2 \%) \\
6(7.2 \%) \\
20(24.1 \%) \\
1(1.2 \%) \\
7(8.4 \%)\end{array}$ & $\begin{array}{c}14(4.8 \%) \\
4(1.4 \%) \\
34(11.8 \%) \\
84(29.1 \%) \\
17(5.9 \%) \\
38(13.1 \%) \\
79(27.3 \%) \\
1(0.3 \%) \\
18(6.2 \%)\end{array}$ & $\begin{array}{c}39(5.5 \%) \\
24(3.4 \%) \\
155(21.8 \%) \\
151(21.3 \%) \\
51(7.2 \%) \\
66(9.3 \%) \\
167(23.5 \%) \\
13(1.8 \%) \\
44(6.2 \%)\end{array}$ \\
\hline $\begin{array}{l}\text { Ingreso a UCI, estancia y mortalidad } \\
\text { Requerimiento de UCI } \\
\text { Días de estancia en UCI, mediana (RIC) } \\
\text { Días de estancia hospitalaria, mediana (RIC) } \\
\text { Mortalidad intrahospitalaria }\end{array}$ & $\begin{array}{c}11(3.3 \%) \\
4.5(4-11.5) \\
6(4-9) \\
14(4.1 \%)\end{array}$ & $\begin{array}{c}2(2.4 \%) \\
5(1-9) \\
7(5-10) \\
1(1.2 \%)\end{array}$ & $\begin{array}{c}10(3.5 \%) \\
5.5(3-10.7) \\
6(4-9) \\
8(2.8 \%)\end{array}$ & $\begin{array}{c}23(3.2 \%) \\
5(3-9) \\
6(4-9) \\
23(3.2 \%)\end{array}$ \\
\hline $\begin{array}{l}\text { Etiología de la descompensación } \\
\text { Arritmias } \\
\text { Enfermedad coronaria } \\
\text { Infección } \\
\text { No adherencia a dieta } \\
\text { No adherencia a tratamiento } \\
\text { Otros } \\
\text { No registrada } \\
\text { Tromboembolia pulmonar }\end{array}$ & $\begin{array}{c}41(12.1 \%) \\
24(7.1 \%) \\
35(10.4 \%) \\
3(0.9 \%) \\
68(20.1 \%) \\
48(14.2 \%) \\
117(34.6 \%) \\
2(0.6 \%)\end{array}$ & $\begin{array}{c}11(13.3 \%) \\
7(8.4 \%) \\
8(9.6 \%) \\
0(0 \%) \\
12(14.5 \%) \\
20(24.1 \%) \\
25(30.1 \%) \\
0(0 \%)\end{array}$ & $\begin{array}{c}26(9 \%) \\
6(2.1 \%) \\
42(14.5 \%) \\
1(0.3 \%) \\
43(14.9 \%) \\
43(14.9 \%) \\
123(42.6 \%) \\
5(1.7 \%)\end{array}$ & $\begin{array}{c}78(11 \%) \\
37(5.2 \%) \\
85(12.0 \%) \\
4(0.6 \%) \\
123(17.3 \%) \\
111(15.6 \%) \\
265(37.3 \%) \\
7(1.0 \%)\end{array}$ \\
\hline
\end{tabular}

FEVI: fracción de eyección del ventrículo izquierdo; RIC: rango intercuartílico; UCI: unidad de cuidados intensivos.

\section{Variables de tratamiento}

Los medicamentos previos al ingreso más usados fueron betabloqueadores $(54.9 \%)$, furosemida $(53.7 \%)$, estatinas (37\%), antiagregantes (38.3\%), antagonistas del receptor de mineralocorticoides (25.3\%), antagonistas del receptor de la angiotensina 2 (ARA 2) (37.5\%), inhibidores de la enzima convertidora de angiotensina (IECA) $(29,2 \%)$ y antagonistas del calcio (21.5\%). En el grupo de FEVI intermedia, el $51.8 \%$ usaba betabloqueadores, el $36.1 \%$ ARA 2 y el $27.7 \%$ IECA; además, el $15.7 \%$ tomaba antagonistas del receptor de mineralocorticoides dentro del manejo de su falla cardiaca, el $39.8 \%$ estaba usando un diurético de asa y el $5.8 \%$ digitálicos.

Al comparar el manejo al ingreso y al egreso, el uso de antagonistas del receptor de mineralocorticoides en Ios pacientes con FEVI intermedia aumentó al $26.5 \%$, el de betabloqueadores al $78.3 \%$, el de diurético de asa al $61.4 \%$ y el de digitálicos al $8.4 \%$. De los pacientes con antagonistas del receptor de mineralocorticoides al egreso, el $77 \%$ los iniciaron durante la hospitalización; tendencia observada también en los pacientes con FEVI reducida (Tabla 4).

\section{Pacientes con ecocardiograma extrahospitalario}

Este grupo tuvo un comportamiento similar al de aquellos pacientes clasificados según la FEVI con estudio intrahospitalario.

\section{Discusión}

En este estudio se describen, por primera vez, las características y el pronóstico de una cohorte de pacientes hospitalizados con falla cardiaca y FEVI en rango intermedio, admitidos en un hospital universitario de cuarto nivel de complejidad. En cuanto al total de la muestra (sin discriminación por FEVI), puede afirmarse que mostró un comportamiento similar al de la cohorte MED-ICA, con epidemiología comparable, aunque con menor mortalidad intrahospitalaria ${ }^{6}$. 
Tabla 4. Variables de tratamiento

\begin{tabular}{|c|c|c|c|c|}
\hline Variable & $\begin{array}{l}\text { FEVI < 40\% } \\
338(39.1 \%)\end{array}$ & $\begin{array}{c}\text { FEVI } 40-49 \% \\
83(9.6 \%)\end{array}$ & $\begin{array}{l}\text { FEVI > 50\% } \\
289(33.4 \%)\end{array}$ & $\begin{array}{c}\text { Total } \\
710(100 \%)\end{array}$ \\
\hline $\begin{array}{l}\text { Medicación al ingreso } \\
\text { Antiagregantes } \\
\text { Anticoagulantes } \\
\text { IECA } \\
\text { ARA } 2 \\
\text { Betabloqueadores } \\
\text { Calcioantagonistas } \\
\text { Antagonistas del receptor de mineralocorticoides } \\
\text { Digitálicos } \\
\text { Estatinas } \\
\text { Tiazidas } \\
\text { Furosemida } \\
\text { Otros }\end{array}$ & $\begin{array}{l}128(37.9 \%) \\
46(13.6 \%) \\
107(31.7 \%) \\
107(31.7 \%) \\
178(52.7 \%) \\
58(17.2 \%) \\
93(27.5 \%) \\
27(8 \%) \\
121(35.8 \%) \\
15(4.4 \%) \\
179(53 \%) \\
186(55 \%)\end{array}$ & $\begin{array}{l}28(33.7 \%) \\
17(20.5 \%) \\
23(27.7 \%) \\
30(36.1 \%) \\
43(51.8 \%) \\
16(19.3 \%) \\
13(15.7 \%) \\
6(7.2 \%) \\
31(37.3 \%) \\
8(9.6 \%) \\
33(39.8 \%) \\
43(51.8 \%)\end{array}$ & $\begin{array}{l}106(36.7 \%) \\
43(14.9 \%) \\
72(24.9 \%) \\
128(44.3 \%) \\
135(46.7 \%) \\
86(29.8 \%) \\
43(14.9 \%) \\
8(2.8 \%) \\
96(33.2 \%) \\
33(11.4 \%) \\
138(47.8 \%) \\
164(56.7 \%)\end{array}$ & $\begin{array}{l}262(36.9 \%) \\
106(14.9 \%) \\
202(28.5 \%) \\
265(37.3 \%) \\
356(50.1 \%) \\
160(22.5 \%) \\
149(21.0 \%) \\
41(5.8 \%) \\
248(34.9 \%) \\
56(7.9 \%) \\
350(49.3 \%) \\
393(55.4 \%)\end{array}$ \\
\hline $\begin{array}{l}\text { Medicación al alta } \\
\text { Antiagregantes } \\
\text { Anticoagulantes } \\
\text { IECA } \\
\text { ARA } 2 \\
\text { Betabloqueadores } \\
\text { Antagonistas del calcio } \\
\text { Antagonistas del receptor de mineralocorticoides } \\
\text { Digitálicos } \\
\text { Estatinas } \\
\text { Tiazidas } \\
\text { Furosemida } \\
\text { Otros }\end{array}$ & $\begin{array}{l}148(43.8 \%) \\
71(21 \%) \\
170(50.3 \%) \\
98(29 \%) \\
272(80.5 \%) \\
36(10.7 \%) \\
198(58.6 \%) \\
49(14.5 \%) \\
158(46.7 \%) \\
3(0.9 \%) \\
246(72.8 \%) \\
208(61.5 \%)\end{array}$ & $\begin{array}{l}32(38.6 \%) \\
30(36.1 \%) \\
39(47.0 \%) \\
30(36.1 \%) \\
65(78.3 \%) \\
15(18.1 \%) \\
22(26.5 \%) \\
7(8.4 \%) \\
43(51.8 \%) \\
2(2.4 \%) \\
51(61.4 \%) \\
51(61.4 \%)\end{array}$ & $\begin{array}{c}85(29.4 \%) \\
57(19.7 \%) \\
74(25.6 \%) \\
113(39.1 \%) \\
149(51.6 \%) \\
64(22.1 \%) \\
40(13.8 \%) \\
4(1.4 \%) \\
110(38.1 \%) \\
15(5.2 \%) \\
169(58.5 \%) \\
183(63.3 \%)\end{array}$ & $\begin{array}{l}265(37.3 \%) \\
158(22.3 \%) \\
283(39.9 \%) \\
241(33.9 \%) \\
486(68.5 \%) \\
115(16.2 \%) \\
260(36.6 \%) \\
60(8.5 \%) \\
311(43.8 \%) \\
20(2.8 \%) \\
466(65.6 \%) \\
442(62.3 \%)\end{array}$ \\
\hline
\end{tabular}

ARA 2: antagonistas del receptor de la angiotensina 2; FEVI: fracción de eyección del ventrículo izquierdo; IECA: inhibidores de la enzima convertidora de angiotensina.

Al presente, son varias las series internacionales que se han esforzado por caracterizar el grupo con FEVI intermedia. En general, se ha encontrado que un 10$25 \%$ de los pacientes con falla cardiaca pueden ser clasificados en este grupo ${ }^{7-11}$. Nuestro estudio demuestra una prevalencia en el límite inferior de este rango, con una edad media que concuerda con la hallada en las demás poblaciones revisadas y que refleja el envejecimiento poblacional. La ligera predominancia del sexo femenino discrepa de lo descrito en la mayoría de las cohortes (CHART-2 ${ }^{8}$, Rastogi et al. ${ }^{9}$, Gómez-Otero et al. $^{10}$, registro RICA ${ }^{11}$, TIME-CHF ${ }^{12}$, Swede $\mathrm{HF}^{13}$, ESC-HF-LT ${ }^{14}$ ), pero sigue una distribución igual a la de registros extensos como OPTIMIZE-HF ${ }^{15}$ y GWTF-HF ${ }^{16}$. Cuando se compara con los grupos de FEVI preservada y reducida en estos datos demográficos, cae en un punto intermedio, hallazgo reforzado en la muestra de este estudio ${ }^{7,11,17}$.

Una observación que concuerda en la mayoría de las series es la presencia de enfermedad coronaria como etiología y comorbilidad de la falla cardiaca en Ios pacientes con FEVI intermedia, elemento que los hace semejantes a aquellos con FEVI reducida. Esta fue la etiología más frecuente en nuestra cohorte, con un porcentaje similar al del grupo de FEVI reducida, pero por debajo de lo encontrado en cohortes como CHART-2 (52.9\%), el registro RICA (38\%) y ESC-HF-LT $(41.8 \%)^{8,11,14}$. Cabe afirmar lo mismo sobre la isquemia miocárdica como comorbilidad en los pacientes evaluados, con una prevalencia cercana a la de quienes tenían FEVI reducida, pero de nuevo menor que en las cohortes internacionales. Algunos autores han sugerido que la alta tasa de enfermedad coronaria podría explicarse por una progresión de FEVI intermedia a reducida después de un evento coronario, o por una recuperación de la función sistólica con el tratamiento ${ }^{7,11}$.

En cuanto a las demás etiologías, la cardiopatía hipertrófica también tuvo una frecuencia cercana a la del grupo de FEVI reducida y mucho menor que la de los pacientes con FEVI preservada. Esto también se ha descrito previamente y hace pensar en una fisiopatología semejante. Llama la atención la baja prevalencia de comorbilidad encontrada en los pacientes de este estudio, muy por debajo de la citada en muestras nacionales e internacionales, lo que hace pensar en una falta de adecuado registro en las historias clínicas e incluso evaluar la posibilidad de un error sistemático, 
no diferencial, en la recolección de datos en este aspecto concreto. En las demás series se han encontrado unas prevalencias de hipertensión arterial y de fibrilación auricular similares a las de los pacientes con FEVI preservada o en un punto intermedio entre la preservada y la reducida ${ }^{7,8,10-15}$.

La disnea fue el síntoma más reportado en nuestra cohorte, seguida del edema, que es la presentación predominante en los demás estudios. Este resultado responde a la epidemiología de la falla cardiaca con manifestaciones congestivas como el perfil más frecuente, sin ser específico de este síndrome ${ }^{6}$.

En esta cohorte, el principal motivo de descompensación fue la falta de adherencia al tratamiento farmacológico, como en MED-ICA, muy superior a la detectada en registros como RICA ${ }^{11}$. Los estudios prospectivos latinoamericanos, como ICARO ${ }^{18}$, BREATHE ${ }^{19}$ y CONAREC XVIIII ${ }^{20}$, evidenciaron que la falta de adherencia farmacológica explica entre el $13 \%$ y el $29 \%$ de las descompensaciones, factor potencialmente modificable o evitable ${ }^{21-23}$.

No hay recomendaciones para el tratamiento de los pacientes con FEVI intermedia, ya que la información sobre su impacto es limitada. Por tanto, se asume que deben manejarse como aquellos con FEVI preservada, con control de su comorbilidad y sintomatología. El uso de diuréticos de asa es similar en todos los grupos, tanto en este como en los demás estudios revisados. Sin embargo, llama la atención el uso de betabloqueadores en un porcentaje cercano al de los pacientes con FEVI reducida, que podría ser reflejo de la indicación por isquemia miocárdica. Además, el uso de antagonistas de la aldosterona se encuentra en un punto medio entre los otros dos grupos, dato que coincide en la mayoría de las cohortes. Los resultados del estudio TOPCAT muestran un mejor pronóstico de los pacientes con FEVI del $45-49 \%$ tratados con espironolactona, pero aún se requieren más luces respecto a la utilidad de la terapia ${ }^{22}$.

La mortalidad intrahospitalaria fue similar a la descrita en las cohortes internacionales, pero menor que en la cohorte MED-ICA ${ }^{6,14,24}$. Recientemente se han formulado cuestionamientos sobre el valor pronóstico de la clasificación que incluye la FEVI intermedia. Muchos estudios prospectivos han intentado dilucidarlo y han obtenido resultados conflictivos ${ }^{21,23,25-27}$; algunos sugieren que su pronóstico es similar al de los pacientes con FEVI preservada ${ }^{27-29}$. Esto destaca la utilidad de un diseño prospectivo en estudios futuros en nuestra población.

\section{Limitaciones}

El carácter retrospectivo del estudio limita el análisis de los datos a profundidad en diversos aspectos, debido a la dificultad en la recolección de información. Se llevó a cabo en un único centro en la ciudad de Medellín, lo que constituye un posible sesgo de referencia que no demuestre, necesariamente, la realidad global de la falla cardiaca aguda en rango intermedio en Colombia. El seguimiento fue a corto plazo y se limitó a la mortalidad intrahospitalaria.

Para clasificar la falla cardiaca según la FEVI del ecocardiograma en los tres grupos (reducida, intermedia y conservada), se utilizaron los registros disponibles en la institución en donde se desarrolló la investigación. Más de la mitad de los pacientes no tenían registro de ecocardiogramas previos y un alto porcentaje solo tenían reportado un valor aislado, sin información sobre el año ni la técnica de medición, razón que obligó a separarlos en el análisis de quienes tenían estudio intrahospitalario. En estudios futuros se podrá evaluar la correlación entre los ecocardiogramas previos a la descompensación y los intrahospitalarios.

También se dificultó la recolección de variables con valor pronóstico, como los péptidos natriuréticos, ya que es una prueba aún poco disponible y utilizada en nuestro medio. Además, cabe mencionar que en este estudio se encontró un subregistro de algunas enfermedades concomitantes, entre ellas la dislipidemia, lo cual probablemente se debe a un sesgo de selección al tratarse de pacientes hospitalizados.

\section{Conclusiones}

La población con falla cardiaca aguda de esta cohorte presenta similitudes con otras poblaciones internacionales y algunas nacionales, pero con una mortalidad y una estancia hospitalaria menores que las descritas en el mismo centro en una cohorte previa, a pesar de la edad, la carga de comorbilidad y la falta de adherencia a la terapia farmacológica. Este subgrupo parece tener un comportamiento intermedio cuando se compara con los otros dos, pero con un perfil etiológico que se asemeja al de los pacientes con FEVI reducida. Cabe destacar la alta tasa de pacientes sin diagnóstico etiológico y la aparente ausencia de consenso en la implementación de las directrices de tratamiento farmacológico según la clasificación por la FEVI. 


\section{Agradecimientos}

Los autores agradecen al Doctor Andrés Zapata Cárdenas, especialista en medicina interna, director del proyecto y docente de la Universidad de Antioquia. Y al Doctor Fabián Jaimes Barragán, especialista en medicina interna y epidemiología, y codirector, por su participación en todo el desarrollo de la investigación y la redacción del manuscrito.

\section{Financiamiento}

El estudio fue financiado en su totalidad por los autores.

\section{Conflicto de intereses}

Los autores declaran que no existen conflictos de intereses.

\section{Responsabilidades éticas}

Protección de personas y animales. Los autores declaran que para esta investigación no se han realizado experimentos en seres humanos ni en animales.

Confidencialidad de los datos. Los autores declaran que han seguido los protocolos de su centro de trabajo sobre la publicación de datos de pacientes.

Derecho a la privacidad y consentimiento informado. Los autores declaran que en este artículo no aparecen datos de pacientes.

\section{Bibliografía}

1. Ponikowski P, Voors AA, Anker SD, Bueno H, Cleland JG, Coats AJ, et al. 2016 ESC Guidelines for the diagnosis and treatment of acute and chronic heart failure: The Task Force for the diagnosis and treatment of acute and chronic heart failure of the European Society of Cardiology (ESC). Developed with the special contribution of the Heart Failure Association (HFA) of the ESC. Eur J Heart Fail. 2016;18:891-975.

2. Cho JH, Choe WS, Cho HJ, Lee HY, Jang J, Lee SE, et al. Comparison of characteristics and 3-year outcomes in patients with acute heart failure with preserved, mid-range, and reduced ejection fraction. Circ J. 2019;83:347-56

3. Groote $P$, Cohen $A$, Juillière $Y$, Damy $T$. New guidelines, new recommendations! But what is really new? A pragmatic interpretation of the 2016 European guidelines for the management of chronic heart failure. Arch Cardiovasc Dis. 2017;110:1-6.

4. Kapoor JR, Kapoor R, Ju C, Heidenreich P, Eaben ZJ, Hernández AF, et al. Precipitating clinical factors, heart failure characterization, and outcomes in patients hospitalized with heart failure with reduced, borderline, and preserved ejection fraction. JACC Heart Fail. 2016;4:464-72

5. Nauta JF, Hummel YM, Van-Melle JP, Van-der-Meer P, Lam CS, Ponikowski P, et al. What have we learned about heart failure with mid-range ejection fraction one year after its introduction? Eur Heart Fail. 2017;19:1569-73.

6. Muñoz-Mejía OE, Sierra-Vargas EC, Zapata-Cárdenas A Isaza-Montoya M, Muñoz-Cifuentes ME, Sánchez-Echavarría JD, et al. Caracterización sociodemográfica y clínica de una población con falla cardiaca aguda: cohorte MED-ICA. Rev Colomb Cardiol. 2018;25:200-8.
7. Hsu JJ, Ziaeian B, Fonarow GC. Heart failure with mid-range (borderline) ejection fraction: clinical implications and future directions. JACC Heart Fail. 2017;5:763-71.

8. Tsuij K, Sakata Y, Nochioka K, Miura M, Yamauchi T, Onose T, et al. Characterization of heart failure patients with mid-range left ventricular ejection fraction — a report from the CHART-2 Study. Eur J Heart Fail. 2017;19:1258-69.

9. Rastogi A, Novak E, Platts AE, Mann DL. Epidemiology, pathophysiology and clinical outcomes for heart failure patients with a mid-range ejection fraction. Eur J Heart Fail. 2017;19:1597-605.

10. Gómez-Otero I, Ferrero-Gregori A, Varela-Román A, Seijas-Amigo J, Pascual-Figal DA, Delgado-Jiménez J, et al. La fracción de eyección intermedia no permite estratificar el riesgo de los pacientes hospitalizados por insuficiencia cardiaca. Rev Esp Cardiol. 2017;70:338-46.

11. Guisado-Espartero ME, Salamanca-Bautista $P$, Aramburu-Bodas O, Conde-Martel $A$, Arias-Jiménez JL, Llàcer-Iborra $P$, et al. Heart failure with mid-range ejection fraction in patients admitted to internal medicine departments: findings from the RICA Registry. Int J Cardiol. 2018;255:124-8.

12. Rickenbacher $P$, Kaufmann BA, Maeder MT, Bernheim A, Goetschalckx K Pfister $\mathrm{O}$, et al. Heart failure with mid-range ejection fraction: a distinct clinical entity? Insights from the Trial of Intensified versus standard Medical therapy in Elderly patients with Congestive Heart Failure (TIME-CHF). Eur J Heart Fail. 2017;19:1586.

13. Savarese G, Vasko P, Jonsson A, Edner M, Dahlström U, Lund LD. The Swedish Heart Failure Registry: a living, ongoing quality assurance and research in heart failure. Ups J Med Sci. 2019;124:65-9.

14. Chioncel O, Chioncel O, Lainscak M, Seferovic PM, Anker SD, Crespo-Leiro MD, et al. Epidemiology and one-year outcomes in patients with chronic heart failure and preserved, mid-range and reduced ejection fraction: an analysis of the ESC Heart Failure Long-Term Registry. Eur J Heart Fail. 2017;19:1574-85.

15. Fonarow GC, Stough WG, Abraham WT, Albert NM, Gheorghiade M, Greenberg $\mathrm{BH}$, et al. Characteristics, treatments, and outcomes of patients with preserved systolic function hospitalized for heart failure: a report from the OPTIMIZE-HF Registry. J Am Coll Cardiol. 2007;50:768-77.

16. Ambardekar AV, Fonarow GC, Hernández AF, Pan W, Yancy CW, Krantz MJ. Characteristics and in-hospital outcomes for nonadherent patients with heart failure: findings from Get With The Guidelines-Heart Failure (GWTG-HF). Am Heart J. 2009;158:644-52.

17. Lopatin $Y$. Heart failure with mid-range ejection fraction and how to treat it. Card Fail Rev. 2018;4:9-13.

18. Castro GP, Vukasovic RJL, Garcés SE, Sepúlveda ML, Ferrada KM, Alvarado OS. Insuficiencia cardiaca en hospitales chilenos: Resultados del Registro Nacional de Insuficiencia Cardiaca. Grupo ICARO. Rev Med Chil. 2004;132:655-62.

19. Albuquerque DC, Souza Neto JD, Bacal F, Rohde LEP, Bernárdez-Pereira S, Berwanger O, et al. I Brazilian Registry of Heart Failure - clinical aspects. Care quality and hospitalization outcomes. Arq Bras Cardiol. 2015;104:433-42.

20. Corradi L, Pérez G, Costabel JP, González N, Da Rosa W, Amirano MA et al. XVIII CONAREC registry on decompensated heart failure in Argentina. Rev Argent Cardiol. 2014;82:494-503.

21. Meta-analysis Global Group in Chronic Heart Failure (MAGGIC). The survival of patients with heart failure with preserved or reduced left ventricular ejection fraction: an individual patient data meta-analysis. Eur Heart J. 2012;33:1750-7

22. Solomon SD, Claggett B, Lewis EF, Desai A, Anand I, Sweitzer NK, et al. TOPCAT Investigators. Influence of ejection fraction on outcomes and efficacy of spironolactone in patients with heart failure with preserved ejection fraction. Eur Heart J. 2016;37:455-62.

23. Senior JM, Lugo LH, Acosta N, Acosta JL, Díaz J, Osío OH, et al. Guía de práctica clínica para el síndrome coronario agudo. Ministerio de Salud y Protección Social. 2012;17:7-43

24. Nadar SK, Tariq $\mathrm{O}$. What is heart failure with mid-range ejection fraction? A new subgroup of patients with heart failure. Card Fail Rev. 2018;4:6-8.

25. Altaie $S$, Khalife $W$. The prognosis of mid-range ejection fraction heart failure: a systematic review and meta-analysis. ESC Heart Fail. 2018:5:1008-16

26. Choi KH, Yeon-Lee G, Choi JO, Jeon ES, Lee HY, Cho HJ, et al. Outcomes of de novo and acute decompensated heart failure patients according to ejection fraction. Heart. 2018;104:525-32.

27. Bhambhani V, Kizer JR, Lima JA, Van-der-Harst P, Bahrami H, Nayor M, et al. Predictors and outcomes of heart failure with mid-range ejection fraction. Eur J Heart Fail. 2018;20:651-9.

28. Bartunek J, Davison B, Sherman W, Povsic T, Henry TD, Gersh B, et al Congestive Heart Failure Cardiopoietic Regenerative Therapy (CHART-1) trial design. Eur J Heart Fail. 2016;18:160-8

29. Shiga T, Suzuki A, Haruta S, Mori F, Ota Y, Yagi M, et al. Clinical characteristics of hospitalized heart failure patients with preserved, mid-range, and reduced ejection fractions in Japan. ESC Heart Fail. 2019:6:475-86. 